\title{
Relación entre el índice gradiente dióxido de carbono/gradiente arteriovenoso de oxígeno y lactato en el choque séptico por neumonía
}

\author{
Washington Alfonso Trujillo-Ulloa* 1, a ; José Elías Cabrejo Paredes 2, b
}

\section{RESUMEN}

Objetivo: Determinar la relación del índice gradiente de dióxido de carbono/gradiente arteriovenoso de oxígeno y lactato en pacientes con neumonía complicada y choque séptico en áreas críticas del Hospital III EsSalud Chimbote durante el periodo 2018 y 2019.

Materiales y métodos: Estudio de tipo cuantitativo, observacional, correlacional y longitudinal de tendencias. La unidad de análisis está constituida por los pacientes con neumonía complicada por choque séptico que presentan lecturas de índice de gradiente dióxido de carbono / gradiente arteriovenoso de oxígeno y lecturas de lactato.

Resultados: Se incluyeron 90 pacientes con neumonía complicada por choque séptico. El presente trabajo de investigación demuestra una fuerte relación entre el índice de gradiente de gases y el lactato sérico: al inicio, 3 horas, 6 horas y a las 12 horas, de iniciado y monitorizado estos pacientes.

Conclusiones: El índice del gradiente de dióxido de carbono/gradiente concentración arterio venoso de oxígeno > 1.4, se relaciona con el lactato sérico, en pacientes con shock séptico por neumonía.

Palabras clave: Dióxido de carbono; Consumo de oxígeno; Choque séptico; Hipoxia de la célula (Fuente: DeCS BIREME).

\section{Relationship between carbon dioxide gradient/arteriovenous oxygen gradient index and lactate in septic shock secondary to pneumonia}

\section{ABSTRACT}

Objective: To determine the relationship between carbon dioxide gradient/arteriovenous oxygen gradient index and lactate in patients with pneumonia complicated by septic shock in areas delivering care for critically ill or injured patients of the Hospital III EsSalud Chimbote between 2018 and 2019.

Materials and methods: A quantitative, observational, correlational, longitudinal trend study. The study population consisted of patients with pneumonia complicated by septic shock who had carbon dioxide gradient/arteriovenous oxygen gradient index and lactate readings.

Results: Ninety (90) patients with pneumonia complicated by septic shock participated in the research. The present study showed a robust relationship between gas gradient index and serum lactate when monitoring patients at baseline, 3 hours, 6 hours and 12 hours.

Conclusions: There is a relationship between carbon dioxide gradient/arteriovenous oxygen gradient index (> 1.4) and serum lactate levels in patients with septic shock secondary to pneumonia.

Keywords: Carbon dioxide; Oxygen consumption; Shock, septic; Cell hypoxia (Source: MeSH NLM).

\footnotetext{
1 Hospital III EsSalud, Unidad de Cuidados Intensivos. Chimbote, Perú.

2 Universidad Nacional de Trujillo, Escuela de Posgrado. Trujillo, Perú.

a Médico intensivista, Doctor en Medicina.

b Profesor Principal del Departamento de Medicina Preventiva, Facultad de Medicina.

*Autor corresponsal
} 


\section{INTRODUCCIÓN}

La neumonía es una de las principales causas de morbimortalidad en la comunidad y ocasiona un importante número de fallecimientos en los hospitales. El agente etiológico más frecuente es el neumococo y se estima que la resistencia de esta bacteria a la penicilina podría encontrarse alrededor del $22 \%{ }^{(1,2)}$.

La sepsis es la afección bacteriana que aparece cuando la respuesta del huésped a una infección causa una disfunción orgánica sistémica en el huésped. Además, sigue predominando como una importante entidad relacionada a morbilidad y mortalidad ${ }^{(3,4)}$, por lo que se convierte en un gran problema de salud en todo el mundo, especialmente, en los países en vías de desarrollo donde impacta seriamente en la salud pública y en la economía ${ }^{(5)}$. De acuerdo a investigaciones recientes, la sepsis contribuye o causa entre un tercio y la mitad de todas las muertes en hospitales de Estados Unidos ${ }^{(6,7)}$.

La sepsis es un cuadro muy frecuente en el área de observación de una emergencia hospitalaria. En las unidades de cuidados intensivos representa, aproximadamente, el $20 \%$ de los motivos de ingreso a esta unidad y, asimismo, continúa siendo la principal causa de muerte a nivel mundial ${ }^{(8)}$. Las estimaciones de la incidencia y el resultado de la sepsis en muchos países desarrollados varían un tanto con respecto a los generados en los Estados Unidos, pero todos los datos disponibles confirman que todavía es un importante problema de salud pública ${ }^{(3)}$. Los factores que aumentan el riesgo de sepsis en los países con ingresos intermedio y bajo son la pobreza, la superpoblación, una atención sanitaria básica inadecuada, programas inadecuados de higiene y salud pública y, en algunas naciones, una prevalencia muy elevada de infección por el virus de la inmunodeficiencia humana $(\mathrm{VIH})$. Una realidad muy cercana es la que describe el estudio de Arroyo Sánchez et al. que reporta 34 \% de incidencia de sepsis en pacientes que ingresaron a $\mathrm{UCI}{ }^{(8)}$.

En los Estados Unidos, entre 1979 y 2000, se identificaron 10319418 casos de sepsis, que fue más común en varones que en mujeres (media anual riesgo relativo 1,28; $95 \%$ intervalo confidencia 1,24 a 1,32) y en las personas de raza no blanca que en las de raza blanca (media anual riesgo relativo 1,90; $95 \%$ intervalo confidencia 1,81 a 2,00). Durante ese periodo también se evidenció un incremento anual de la incidencia de sepsis, que pasó de 8,7 \% (aproximadamente, 164000 casos o 82,7 por cada 100000 habitantes) a cerca de 660000 casos $(240,4$ por cada 100000 habitantes).

Durante el periodo 1979 a 1984, la tasa de mortalidad cayó de $27,8 \%$ a $17,9 \%$ con predominio en la población de raza negra ${ }^{(5,9)}$. En el año 2001, Angus et al. utilizaron datos administrativos para estimar que hubo 751000 casos (3,0 por 1000 habitantes) de sepsis en los Estados Unidos anualmente, de los cuales la mitad fueron admitidos a la Unidad de Cuidados Intensivos ( $\mathrm{UCl}$ ), lo que incrementó en promedio 20 días la estancia hospitalaria, y elevó de manera significativa los costos de atención: USD 22 100 por caso y USD 16500 millones de gasto anual en ese país (9), y así conllevó a más de 200000 muertes por año. De otro lado, en Australia, durante los años 2007 y 2008, la incidencia de sepsis fue, 1180 por cada 100000 habitantes, aproximadamente ${ }^{(3,10)}$.

A inicios del siglo veinte, se realizaron estudios prospectivos sobre la mortalidad por sepsis en el mundo. En ellos, las tasas fueron muy variables entre cada país; por ejemplo, la mortalidad en Brasil fue del 46,9 \%; en Noruega, del $27 \%$; en Australia, de 37,5\%; y en Francia, 41,9\%. Durante el año 2003, en Europa, Canadá e Israel la mortalidad osciló entre el 19,6 \% y 49,3\%; en Estados Unidos alcanzó el 47,3\%; finalmente, en Pakistán se aproximó al $90 \%$; estas variaciones dependen de la velocidad y severidad de la instalación de la injuria ${ }^{(3,8)}$. Asimismo, el cuadro de sepsis influye en los altos costos hospitalarios, estadías hospitalarias prolongadas, deficiente calidad de vida de los pacientes al alta de la unidad de cuidados intensivos, etc. ${ }^{(5)}$.

A pesar del avance de las últimas décadas en el manejo de pacientes críticos con cuadros de sepsis y choque séptico, la alta mortalidad (alrededor del $30 \%$ ) sigue predominando de manera invariable, con una tendencia a incrementarse, lo que ha motivado la expectativa de los expertos mundiales por el mejoramiento de la "Campaña de Sobrevivencia a la Sepsis" (4). Hoy en día, está vigente la guía del año 2016 que pone énfasis en la reanimación protocolizada y cuantitativa de pacientes con hipoperfusión tisular inducida por sepsis (choque séptico). Dichas conductas terapéuticas deben iniciarse tan precozmente como se reconozca el estado de hipoperfusión y sin retrasar el ingreso a la unidad de cuidados intensivos ${ }^{(11)}$.

La investigación prospectiva observacional realizada en la Unidad de Cuidados Intensivos del Hospital Universitario de la Pontificia Universidad Católica de Chile (julio de 2011 a noviembre de 2012) consistió en determinar el momento para la suspensión la resucitación por fluidos. Tuvo como determinantes terapéuticos a la normalización de la saturación venosa central de oxígeno $\left(\mathrm{ScvO}_{2}\right)$ y/o lactato. Intervinieron 104 pacientes con choque séptico con hiperlactatemia, con mediciones al inicio, a las 2, 6 y 24 horas del manejo en $\mathrm{UCl}$, y los resultados fueron los siguientes: a las 6 horas de iniciado el manejo en $\mathrm{UCl}$, los niveles de $\mathrm{ScVO}_{2}$, gradiente arteriovenoso central de $\mathrm{PCO}$, el lactato se normalizaron de manera más lenta comparado con la línea base $(4,0 \mathrm{mmol}$ vs $2,7 \mathrm{mmol}, p<0,01)$, y solo el $52 \%$ de los pacientes alcanzaron la normalidad a las 24 
horas del tratamiento ${ }^{(12)}$.

El manejo del choque séptico se basa en lograr alcanzar, en el menor tiempo posible, niveles de presión venosa central (PVC) de 8 a $12 \mathrm{mmHg}$, presión arterial media (PAM) $\geq 65 \mathrm{~mm} \mathrm{Hg}$, diuresis $\geq 0,5 \mathrm{ml} / \mathrm{kg} /$ hora, saturación venosa central $\left(\mathrm{ScvO}_{2}\right)>70 \%$ y normalización del lactato sérico $<4 \mathrm{mmol} / \mathrm{L}$. Estos objetivos logrados muchas veces no reflejan el constante desequilibrio celular entre el aporte y la demanda de oxígeno, y en consecuencia la hipoperfusión se mantiene. ${ }^{(13,14)}$.

En la última década, el análisis de los gases arteriales muestra efectos beneficiosos en la evaluación de la adecuada perfusión tisular durante el choque séptico ${ }^{(15)}$. En el contexto un cuadro refractario a todo tipo de intervención terapéutica, los estudios evidencian que existe una buena correlación entre el desequilibrio de la presión venosa de $\mathrm{CO}_{2}$ (estado de hipercapnia venosa) y el estado de perfusión tisular, debido al aumento del gradiente venoarterial del $\mathrm{CO}_{2}$ y por lo tanto, a la reducción del flujo sanguíneo sistémico y pulmonar; y posteriormente, la manifestación de los efectos deletéreos para la evolución del paciente ${ }^{(16-18)}$.

Los resultados que arroje este proyecto tendrán impacto en la identificación precoz del estado de hipoperfusión tisular, con la consecuente mejora en la morbimortalidad de los pacientes, en el tiempo de estancia hospitalaria, en la reducción de costos de hospitalización, en el tiempo del soporte ventilatorio como coadyuvante del manejo del shock séptico y en su repercusión en la evolución del paciente ${ }^{(19)}$.

Uno de los primeros enfoques mundialmente protocolizados en el manejo del choque séptico es la obtención de la $\mathrm{ScvO}_{2}$ por encima del $70 \%$; sin embargo, no parecería ser un fuerte indicador para determinar el equilibrio entre el aporte de oxígeno y la demanda catabólica celular $(20,21)$. Por ello es necesario de determinar cuál es la relación entre el índice gradiente del dióxido de carbono/concentración arteriovenosa de oxígeno y el lactato en el choque séptico por neumonía en pacientes de áreas críticas del Hospital III EsSalud Chimbote durante los años 2018 y $2019^{(18)}$.

\section{MATERIALES Y MÉTODOS}

\section{Diseño y población de estudio}

El estudio, según enfoque, es tipo analítico, prospectivo, observacional, correlacional y longitudinal de tendencias. La investigación se realizó en las áreas críticas (Emergencia y Cuidados Intensivos) durante los años 2018 y 2019. La unidad de análisis son los pacientes con diagnóstico de neumonía que se complicaron con shock séptico, que presentaron lecturas de índice de gradiente dióxido de carbono / gradiente arteriovenoso de oxígeno y lecturas de lactato en el servicio de áreas críticas. En la presente investigación no se consideró el tamaño muestral, ya que se trabajará con toda la población objetivo que asciende a 90 pacientes. En el estudio se incluyeron a los pacientes con edad comprendida entre los 50 y 70 años, que tenían el diagnóstico clínico, gasométrico, radiológico $\mathrm{y} / \mathrm{o}$ tomográfico de neumonía, con acceso venoso central (catéter venoso central) o línea arterial, con saturación venosa central $\left(\mathrm{SvCO}_{2}\right)>70 \%$ y signos de hipoperfusión como presión arterial media (PAM) $\triangle 65 \mathrm{~mm} \mathrm{Hg}$, fiebre $\left(>38,3{ }^{\circ} \mathrm{C}\right)$, hipotermia $\left(<36{ }^{\circ} \mathrm{C}\right)$, frecuencia cardiaca $>90$ latidos por minuto, frecuencia respiratoria > 20 minutos, estado mental alterado, diuresis $<0,5 \mathrm{ml} / \mathrm{kg} / \mathrm{hora}$, hiperglucemia $>140 \mathrm{mg} / \mathrm{dL}$ (en ausencia de diabetes). Se excluyeron a los pacientes con otra etiología de choque (distinta a la séptica de origen respiratorio), y que presentaban hiperglicemia o hipoglicemia, urea $>40$ o creatinina $>1,2$.

\section{Variables y mediciones}

Como variables del estudio se consideraron dos, la primera fue el lactato sérico que es un producto del metabolismo celular, como resultado de la conversión de los alimentos en energía. Es un marcador estandarizado de severidad en el estado de septicemia. Durante el metabolismo aeróbico, el glucógeno se descompone hacia piruvato, con la consecuente producción de energía. En caso de hipercatabolia (sepsis, ejercicio) la degradación del piruvato no es posible y este se convierte en lactato. Refleja la persistencia del metabolismo anaeróbico y el estado de perfusión celular. El indicador de esta variable es $>2 \mathrm{mmol} / \mathrm{L}$ y se emplea la escala nominal. La segunda variable fue el índice gradiente de dióxido de carbono/gradiente de oxígeno. El gradiente de $\mathrm{CO}_{2}$ está en equilibrio con el gradiente de la concentración arteriovenosa de oxígeno. Un valor de $\mathrm{DCO}_{2}$ superior a 2-6 $\mathrm{mmHg}$ muestra un estado de hipoperfusión. A pesar de tener un valor normal de $\mathrm{SvcO}_{2}>70 \%$, todavía es necesario

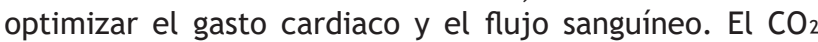
producido a nivel celular en condiciones de metabolismo anaeróbico, se estanca y acumula en el lado venoso post tisular pre capilar alveolar como consecuencia de un gasto cardiaco inadecuado. Esta variable refleja el estado de perfusión celular y se emplea la escala nominal.

Para calcular este valor se aplica la fórmula:

$$
\underset{\triangle \mathrm{C}(\mathrm{a}-\mathrm{v}) \mathrm{O}_{2}}{\triangle \mathrm{PCO}_{2}}>1,4
$$

A los pacientes que ingresan al servicio de áreas críticas del Hospital III EsSalud Chimbote con el diagnóstico de choque séptico por neumonía se les colocan dispositivos de monitoreo hemodinámico: electrodos para registro de funciones vitales (frecuencia cardiaca, frecuencia 
respiratoria, saturación oxígeno, temperatura corporal), una línea arterial para medición constante de presión arterial invasiva, presión arterial de oxígeno $\left(\mathrm{PaO}_{2}\right)$ y presión arterial de $\mathrm{CO}_{2}\left(\mathrm{PaCO}_{2}\right)$, y un catéter venoso central para medición de presión venosa de oxígeno $\left(\mathrm{PvO}_{2}\right)$ y presión venosa de $\mathrm{CO}_{2}\left(\mathrm{PvCO}_{2}\right)$ mediante procesamiento laboratorial de gases arteriales. Así también se realizarán cálculos de medición hemodinámica: concentración arterial de oxígeno $\left(\mathrm{CaO}_{2}\right)$, concentración venosa de oxígeno $\left(\mathrm{CVO}_{2}\right)$, gradiente concentración arteriovenoso de oxígeno $\left(\triangle \mathrm{C}(\mathrm{a}-\mathrm{v}) \mathrm{O}_{2}\right)$ y gradiente presión arteriovenoso de $\mathrm{CO}_{2}\left(\triangle \mathrm{P}(\mathrm{a}-\mathrm{v}) \mathrm{CO}_{2}\right)$. Dichos parámetros laboratoriales y sus cálculos se realizarán al ingreso (T0), a las 3 horas (T3), 6 horas (T6), 12 horas (T12), 24 horas (T24), 48 horas (T48). Los investigadores emplearon el siguiente flujograma:

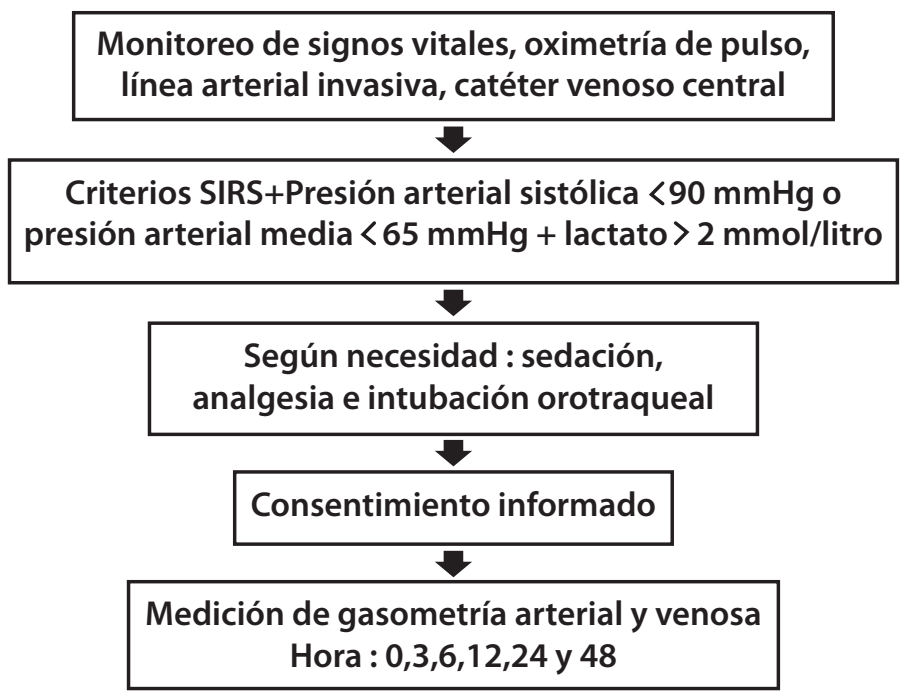

\section{Análisis estadístico}

Para el análisis de datos se utilizó la técnica de la estadística descriptiva que permite determinar el índice gradiente dióxido de carbono / gradiente concentración arteriovenosa de oxígeno, presentarlo según su promedio y desviación estándar; además, el análisis de frecuencias permitirá categorizar esta variable en $\geq 1,4$ y $<1,4$. La estadística descriptiva también permite determinar el nivel de lactato sérico y presentarlo según su promedio y desviación estándar; también se categorizará para los grupos de seguimiento según $>2 \mathrm{mmol} / \mathrm{l}$.

Para relacionar el índice gradiente dióxido de carbono/ gradiente concentración arteriovenoso oxígeno y el lactato, en la forma cuantitativa se utilizará el coeficiente de correlación de Pearson y en el proceso de categorización de variables se utilizará la prueba de chi cuadrado. Según los resultados se puede aplicar el análisis ROC para ver si el índice gradiente dióxido de carbono / gradiente concentración arteriovenosa oxígeno es predictor de un lactato sérico mayor de $2 \mathrm{mmol} / \mathrm{l}$, así mismo se puede obtener el punto de corte para el índice gradiente dióxido de carbono / gradiente concentración arteriovenosa de oxígeno y aplicar las clásicas pruebas diagnósticas de sensibilidad, especificidad, teniendo como prueba de oro a la persistencia de lactato sérico $>2 \mathrm{mmol} / \mathrm{l}$. Todas estas pruebas se realizarán con una confiabilidad del $95 \%$ $(a<0,05)$. Para el procesamiento de esta información se utilizará el programa SPSS (versión 25).

\section{Consideraciones éticas}

El protocolo de investigación fue evaluado por el Comité Institucional de Ética en Investigación de la Facultad de Medicina de la Universidad Nacional de Trujillo, según oficio $\mathrm{N}^{\circ}$ 021-2018-UNT-FM-C.E. Se consideró la firma del consentimiento informado de parte del paciente o del familiar directo. Según el tipo de investigación, no implica personas en situación de vulnerabilidad.

En la presente investigación se aplicaron los siguientes principios éticos ${ }^{(22)}$ :

a. Autonomía: Mediante la firma del consentimiento informado, los familiares de pacientes serán informados de los objetivos, importancia y otros datos necesarios para la toma de decisión, respetando su aceptación o no para su participación.

b. Confidencialidad y anonimato: La información que se obtendrá de los pacientes será estrictamente para fines 
académicos y científicos, los resultados se presentaron de manera global lo que garantiza su anonimato.

c. Beneficencia y no maleficiencia: El estudio se centra en la recolección de datos, sin embargo, los resultados contribuirán al manejo de este tipo de pacientes, lo que abre un campo para que el personal de salud mejore sus estrategias en el manejo de este tipo de pacientes y contribuya a la mejora del estado de salud.

\section{RESULTADOS}

En el estudio se incluyeron 90 pacientes. En la tabla 1 se presenta la distribución, según sexo, de los pacientes con choque séptico de las áreas críticas del Hospital III EsSalud Chimbote durante los años 2018 y 2019. Según la estadística descriptiva, existe una proporcionalidad en la distribución según sexo.

Tabla 1. Pacientes con shock séptico por neumonía, según sexo

\begin{tabular}{|lcc|}
\hline Edad & Masculino & Femenino \\
\hline Promedio & 60,42 & 59,83 \\
\hline Desviación estándar & 5,86 & 6,13 \\
N & 48 & 42 \\
\hline
\end{tabular}

Fuente: Datos recolectados durante la investigación.

En la tabla 2 se muestra la variable índice gradiente dióxido de carbono/gradiente concentración arteriovenosa de oxígeno en pacientes con choque séptico de las áreas críticas del Hospital III EsSalud Chimbote durante los años 2018 y 2019, según datos estadísticos descriptivos en diferentes momentos, desde el inicio hasta los valores registrados a las 48 horas. Encontramos que los valores de la media del índice resultan positivos desde el inicio de la instalación del choque séptico por neumonía, con la tendencia a normalizarse en el transcurrir de las horas siguientes.

Tabla 2. Índice gradiente dióxido de carbono/gradiente concentración arteriovenoso de oxígeno, en pacientes con shock séptico por neumonía

\begin{tabular}{|c|c|c|c|c|c|c|}
\hline \multicolumn{7}{|c|}{$\begin{array}{l}\text { Índice gradiente dióxido de carbono/gradiente concentración } \\
\text { arteriovenoso de oxígeno }\end{array}$} \\
\hline & Inicio & 3 horas & 6 horas & 12 horas & 24 horas & 48 horas \\
\hline Media & 1,86 & 1,63 & 1,31 & 1,02 & 0,97 & 1,76 \\
\hline Mediana & 1,80 & 1,60 & 1,20 & 0,90 & 0,91 & 1,40 \\
\hline Mín & 0,48 & 0,03 & 0,60 & 0,40 & 0,50 & 0,40 \\
\hline Máx & 3,20 & 3,10 & 3,10 & 1,80 & 1,80 & 4,10 \\
\hline Desviación estándar & 0,60 & 0,52 & 0,39 & 0,30 & 0,30 & 1,14 \\
\hline
\end{tabular}

Fuente: Datos recolectados durante la investigación

Esta misma variable (índice gradiente dióxido de carbono/ gradiente concentración arteriovenosa de oxígeno) se muestra en la figura 1: gráfico box-plot (cajas y bigotes), mediante la demostración de la distribución de los valores del índice de gases, en la cual en la hora inicial tienen una mejor tendencia a ubicarse en el rango entre 3,30 y 0,50. Asimismo, muestra la misma tendencia en la disminución de los valores hasta las 48 horas, en la que estos valores empiezan a dispersarse en mayor proporción. 


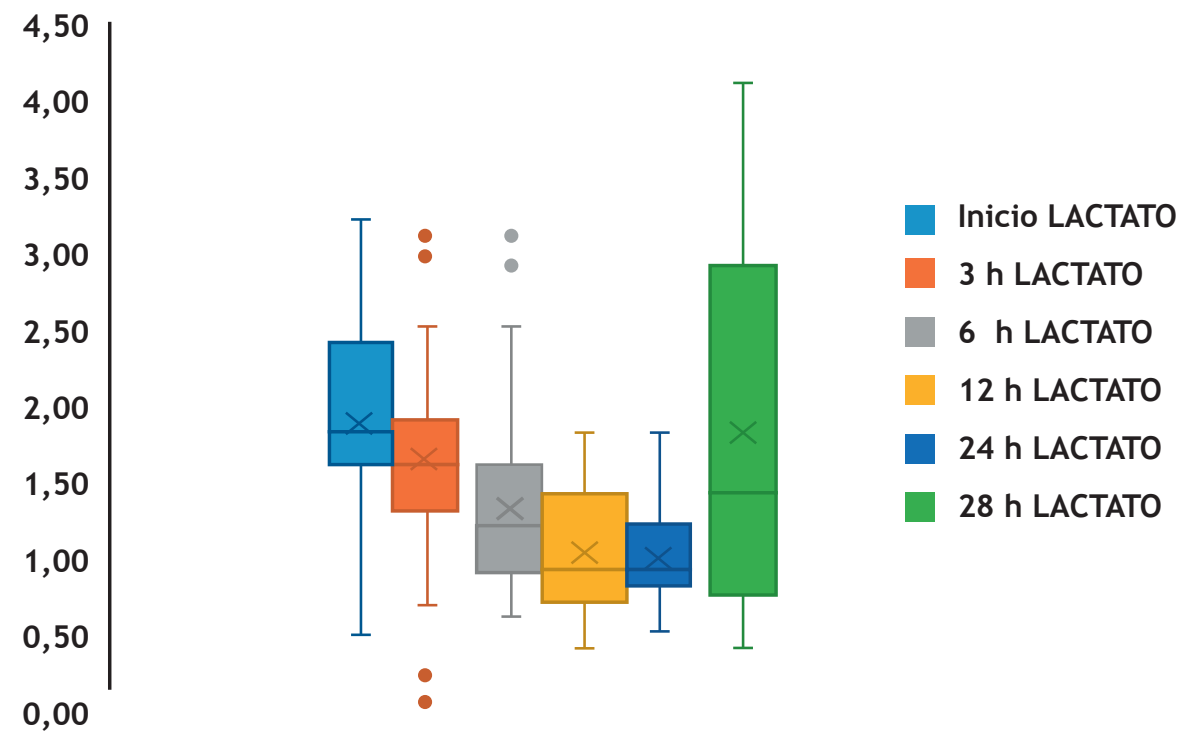

Figura 1. Box-plot para el índice gradiente dióxido de carbono/gradiente concentración arteriovenosa de oxígeno, según evolución en horas

En la tabla 3 se muestra la variable del nivel de lactato en pacientes con choque séptico de las áreas críticas del Hospital III EsSalud Chimbote durante los años 2018 y 2019, según datos estadísticos descriptivos en diferentes momentos, desde el inicio hasta los valores registrados a las 48 horas. Encontramos que los valores de la media del nivel de lactato resultan positivos desde el inicio del choque séptico por neumonía y se mantienen durante las primeras 6 horas. A partir de las 12 horas, estos valores tienden a normalizarse.

Tabla 3. Nivel de lactato sérico, en pacientes con shock séptico por neumonía

\begin{tabular}{lcccccc}
\multicolumn{7}{c}{ Nivel de lactato sérico } \\
& Inicio & 3 horas & 6 horas & 12 horas & 24 horas & 48 horas \\
Media & 2,95 & 2,48 & 2,21 & 1,72 & 1,49 & 1,52 \\
Mediana & 3,10 & 2,40 & 2,20 & 1,70 & 1,40 & 1,40 \\
Mín & 0,80 & 1,10 & 1,10 & 0,90 & 0,50 & 0,40 \\
Máx & 4,10 & 4,60 & 4,43 & 2,80 & 2,20 & 3,90 \\
Desviación estándar & 0,80 & 0,69 & 0,65 & 0,51 & 0,45 & 0,71
\end{tabular}

Fuente: Datos recolectados durante la investigación

En cada momento de la evolución del shock séptico por neumonía, los valores de lactato sérico tienen la misma tendencia de positividad en sus valores, con una distribución proporcional. A partir de las 12 horas empiezan a declinar los valores hasta llegar a valores normales de lactato sérico, según la figura 2: gráfico box-plot (cajas y bigotes). 


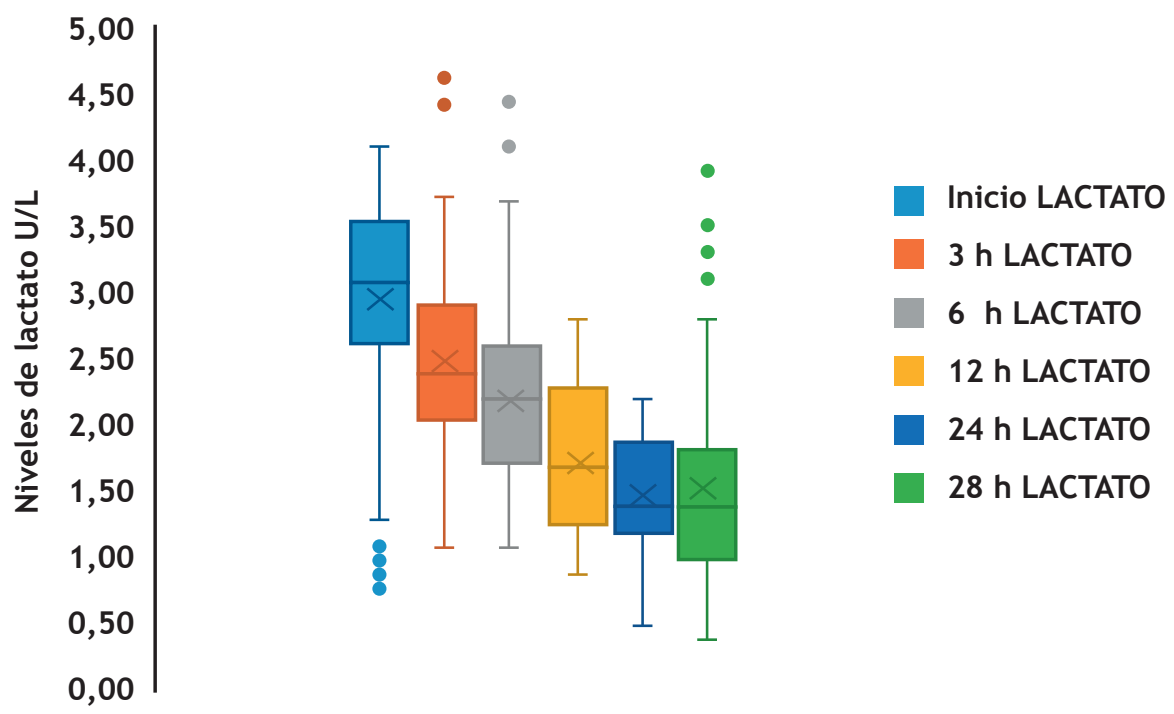

Figura 2. Box-plot para los niveles de lactato sérico, según evolución en horas

Para poder establecer la relación entre el índice gradiente dióxido de carbono / gradiente concentración arteriovenosa de oxígeno con el lactato sérico en pacientes con shock séptico de las áreas críticas del Hospital III
EsSalud Chimbote durante los años 2018 y 2019 se utilizó la correlación de Pearson. En la tabla 4, se muestra la significancia estadística entre las 2 pruebas, lo que hace pensar en la gran relación que existe entre ambas.

Tabla 4. Correlación entre el índice gradiente dióxido de carbono/gradiente concentración arteriovenosa oxígeno vs. lactato sérico, en pacientes con shock séptico por neumonía

\begin{tabular}{lccccccc} 
& Inicio & 3 horas & 6 horas & 12 horas & 24 horas & 48 horas \\
\hline r de Pearson & 0,8178 & 0,6244 & 0,7821 & 0,6791 & 0,6608 & 0,5002 \\
Significancia bilateral & 0,00 & 0,02 & 0,02 & 0,01 & 0,02 & 0,01
\end{tabular}

Fuente: Datos recolectados durante la investigación

En la figura 3 (gráfico de dispersión) se representa la tendencia lineal de la relación entre el índice gradiente dióxido de carbono / gradiente concentración arteriovenosa de oxígeno y lactato, según :tiempo : A (inicio), B (3 horas), C (6 horas), D (12 horas), E (24 horas) y F (48 horas).

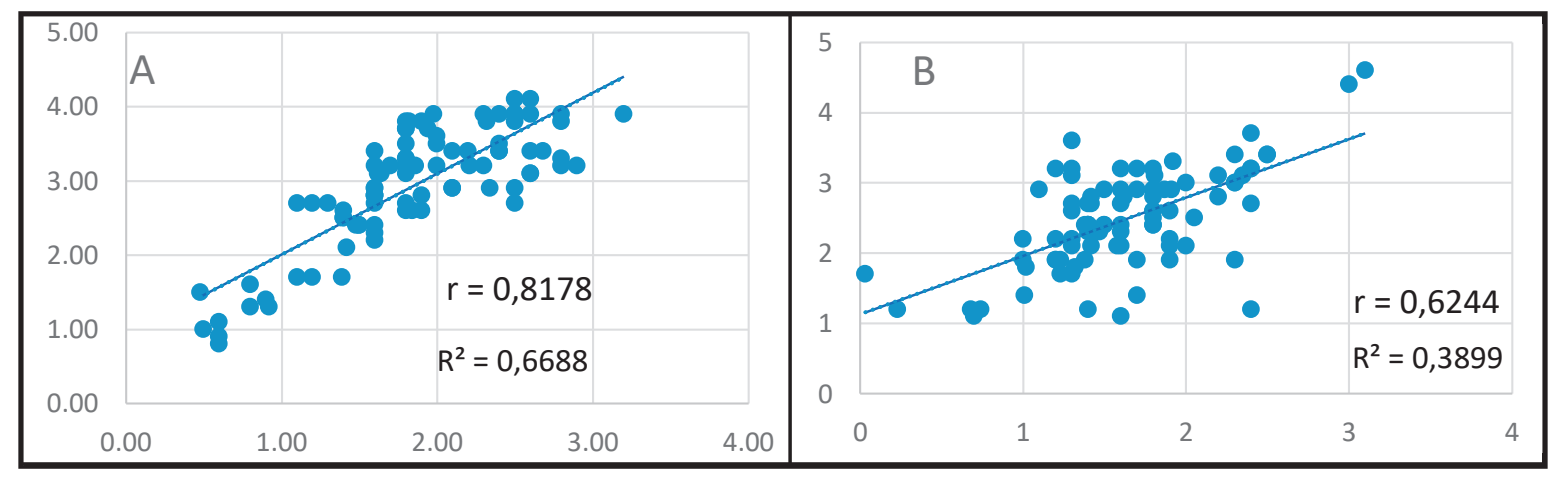


Relación entre el índice gradiente dióxido de carbono/gradiente arteriovenoso de oxígeno y lactato en el choque séptico por neumonía

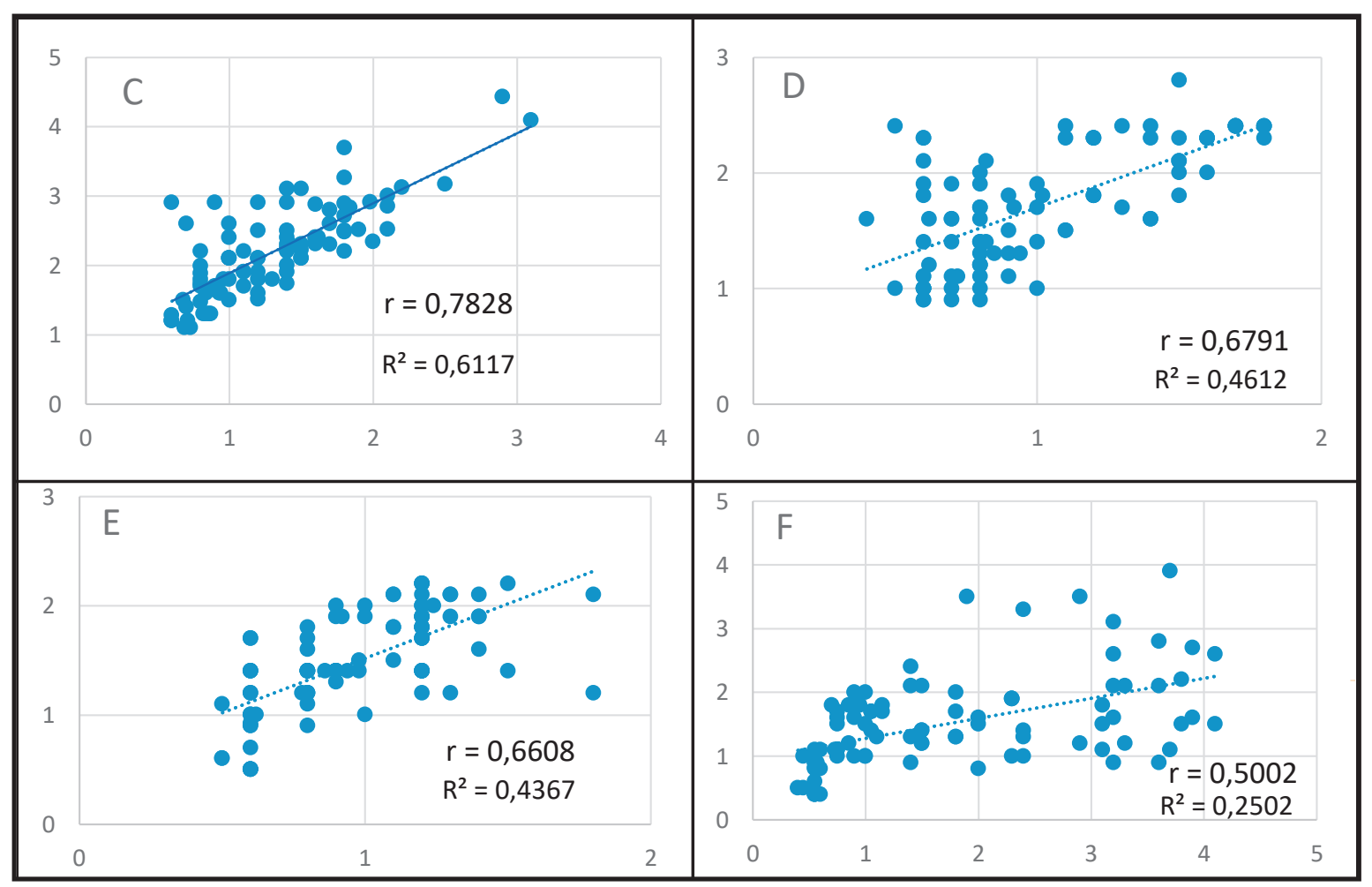

Figura 3. Relación entre el índice gradiente dióxido de carbono / gradiente concentración arteriovenosa de oxígeno y lactato, según tiempo

Para evidenciar si el índice gradiente dióxido de carbono/ gradiente concentración arteriovenosa de oxígeno es un buen predictor del estado del choque séptico por neumonía, según el tiempo evolutivo en horas (A al inicio, $\mathrm{B}$ a las 3 horas, $\mathrm{C}$ a las 6 horas, $\mathrm{D}$ a las 12 horas, $\mathrm{E}$ a las 24 horas y $\mathrm{F}$ a las 48 horas), se utilizó el gráfico de la curva
ROC (en la cual, mientras más se aproxime a la unidad, es mejor predictor). Se encontró que el valor de la curva ROC al inicio fue de 0,995; a las 3 horas, 0,780; a las 6 horas, 0,892 ; a las 12 horas, 0,825 ; a las 24 horas, 0,848 ; y a las 48 horas, de 0,848 .
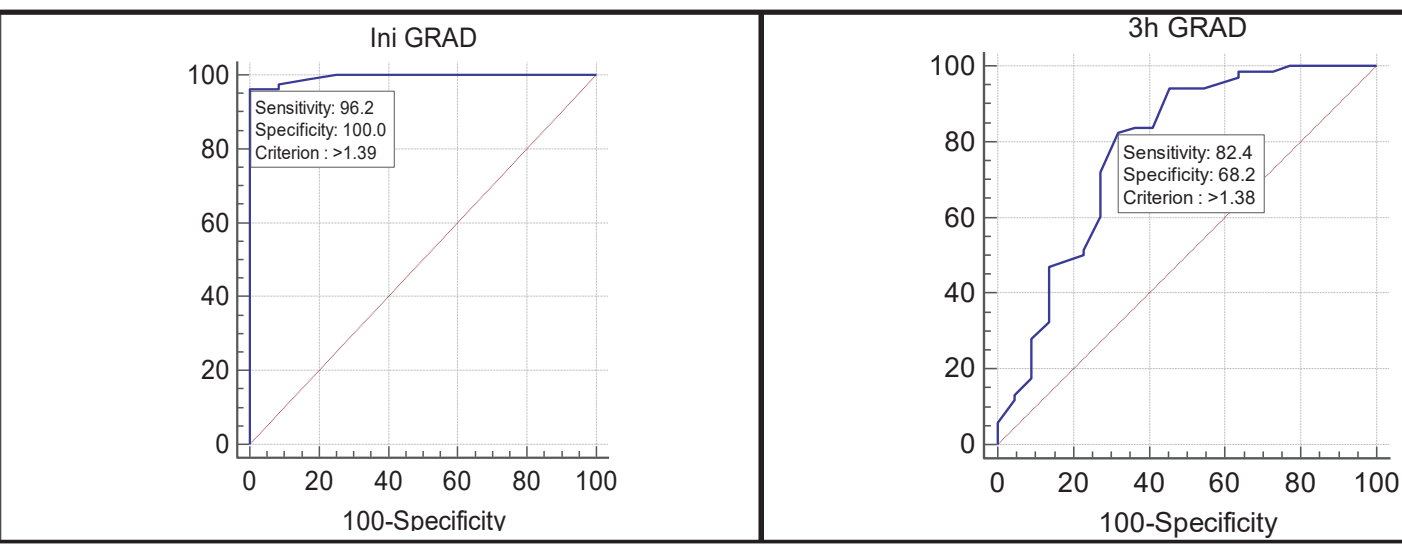


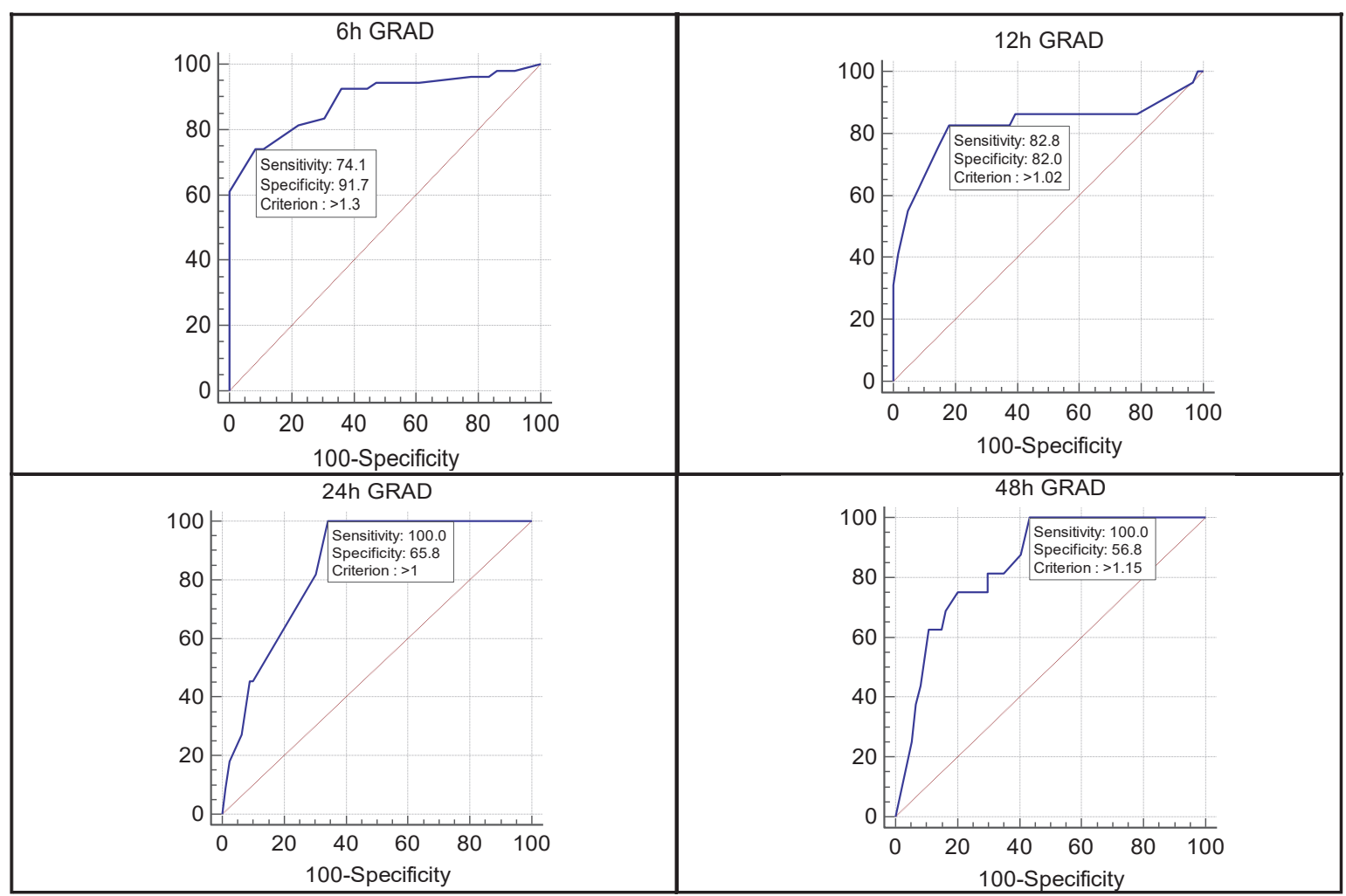

Figura 4. Gráfico de curva ROC

\section{DISCUSIÓN}

La hipoperfusión tisular se produce debido al desequilibrio entre el aporte y el consumo de oxígeno lo cual conlleva a estados de hipoxemia e hipercapnia a nivel tisular, lo que es un factor determinante en la evolución del shock séptico. La importancia del dosaje del índice de gases radica en la contribución con el monitoreo hemodinámico del estado de hipoperfusión, ya que no es suficiente tener valores de presión arterial dentro de parámetros normales debido a que, concomitantemente, puede persistir aún el estado de metabolismo anaeróbico. En nuestro estudio encontramos que durante el estado de hipoperfusión tisular, los niveles del índice de gradiente de $\mathrm{CO}_{2} / \mathrm{O}_{2}$ tuvieron tanta sensibilidad como el dosaje de lactato sérico. Esto está refrendado por el estudio de Hernández Luna en el cual niveles altos de $\mathrm{CO}_{2}$, por encima de $6 \mathrm{mmHg}$, valoran el estado de perfusión tisular; asimismo, estos niveles elevados posteriores a la reanimación predicen un mal pronóstico en 65 pacientes con choque séptico (22). Por otro lado, en el estudio de Hinestrosa et al. encontraron una asociación lineal negativa significativa entre el índice cardiaco y el delta del $\mathrm{CO}_{2}$ que, incluso, estaba mayormente relacionada a mortalidad ${ }^{(23)}$.

El índice de gradiente dióxido de carbono/gradiente concentración arteriovenosa de oxígeno fue un buen predictor del choque séptico a las $3,6,12,24$ y 48 horas, con áreas bajo la curva de ROC de 78,0 89,2; 82,5; 84,8 y 84,8, respectivamente. Los puntos de corte para el gradiente para un lactato sérico > 2 fueron los siguientes: > de 1, 15 (a las 3 horas con sensibilidad de 100 y especificidad de 56,8 ); a las 6 horas se obtuvo $>1,3$ (sensibilidad de 74,1 y especificidad de 91,7 ), a las 12 horas fue $>1,02$ (sensibilidad de 82,8 y especificidad de 82,0 ), a la 24 horas se obtuvo un valor > 1,0 (sensibilidad de 100 y especificidad de 65,8), finalmente, a las 48 horas el valor fue $>1,15$ (sensibilidad de 100 y especificidad de 56,8 ).

Concluimos que la media de los valores de índice del gradiente de dióxido de carbono/gradiente concentración arteriovenoso de oxígeno por encima de 1,4 aparecen al inicio del reconocimiento del choque séptico por neumonía y disminuyen sus niveles a partir de la sexta hora. Además, los niveles de lactato sérico se volvieron positivos (>2 mmol/l) desde el inicio del monitoreo. Finalmente, existió una relación entre el índice del gradiente de dióxido de carbono/gradiente concentración arteriovenosa de oxígeno con el lactato sérico, durante los tiempos de dosaje de ambas pruebas de laboratorio.

Como en todo estudio encontramos ciertas limitaciones, como en el compromiso del personal de las áreas críticas 
del Hospital III EsSalud Chimbote y en el llenado de la ficha de recolección de datos. Asimismo, se recomienda emplear el índice de gradiente de dióxido de carbono/gradiente concentración arteriovenosa de oxígeno por encima de 1,4 en las Unidades de Cuidados Intensivos debido a la casuística de pacientes con choque séptico por neumonía.

Contribuciones de los autores: Todos los autores han realizado conjuntamente, y a partes iguales, la argumentación y la redacción del artículo.

Fuentes de financiamiento: Este artículo ha sido financiado por los autores.

Conflictos de interés: Los autores declaran no tener ningún conflicto de interés.

\section{REFERENCIAS BIBLIOGRÁFICAS}

1. Organización Panamericana de la Salud. Guía de Práctica Clínica: Neumonía adquirida en la comunidad en adultos. 2009. Disponible en: http://bvs. minsa.gob.pe/local/minsa/2418.pdf

2. Mandell L, Wunderink R, Anzueto A, Bartlett J, Campbell G, Dean $\mathrm{N}$, et al. Infectious Diseases Society of America/American Thoracic Society Consensus guidelines on the management of communityacquired pneumonia in adults. Clin Infect Dis. 2007; 44(Suppl. 2): S27-72.

3. Finfer S, Machado FR. The global epidemiology of sepsis, does it matter that we know so little?. Am J Respir Crit Care Med. 2016; 193(3): 228-30.

4. Rhodes A, Evans LE, Alhazzani W, Levy MM, Antonelli M, Ferrer $R$, et al. Surviving Sepsis Campaign: International guidelines for management of sepsis and septic shock. Intensive Care Medicine. Springer Berlin Heidelberg; 2017. pp. 304-77.

5. Mayorga Espichán M. Estrategias para mejorar la sobrevivencia de los pacientes con sepsis severa. Acta Med Perú. 2010; 27(4): 302-9.

6. Dombrovskiy V, Martin A, Sunderram J, Paz H. Rapid increase in hospitalization and mortality rates for severe sepsis in the United States: a trend analysis from 1993 to 2003. Crit Care Med. 2007; 35(5): 1244-50.

7. ProCESS Investigators, Yealy DM, Kellum JA, Huang DT, Barnato AE, Weissfeld LA, et al. A randomized trial of protocol-based care for early septic shock. New Engl J Med. 2014; 370(18): 1683-93.

8. Arroyo Sánches A, Camacho Cosavalente L. Trastornos ácido-base en la Unidad de Cuidados Intensivos del Hospital Víctor Lazarte Echegaray: incidencia, estancia hospitalaria y mortalidad. Acta Med Perú. 2007; 24(2): 91-5.

9. Martín GS, Mannino DM, Eaton S, Moss M. The epidemiology of sepsis in the United States from 1979 through 2000. New Engl J Med. 2003; 348(16): 1546-54.

10. Angus DC, Pereira CA, Silva E. Epidemiology of severe sepsis around the world. Endocr Metab Immune Disord Drug Targets. 2006; 6(2): 207-12.

11. Quenot JP, Binquet C, Kara F, Martinet O, Ganster F, Navellou JC, et al. The epidemiology of septic shock in Intensive Care Units: the prospective multicenter cohort EPISS Study. Crit Care. 2013; 17(2): R65.

12. Hernández G, Luengo C, Bruhn A, Kattan E, Friedman G, OspinaTascon GA, et al. When to stop septic shock resuscitation: clues from a dynamic perfusion monitoring. Ann Intensive Care. 2014; 4(1): 1-9.
13. Ariza M, Gothard J, Macnaughton P, Hooper J, Morgan C, Evans T. Blood lactate and mixed venous-arterial PCO2 gradient as indices of poor peripheral perfusion following cardiopulmonary bypass surgery. Intensive Care Med. 1991; 17(6): 320-4.

14. Takami Y, Masumoto H. Mixed venous-arterial $\mathrm{CO} 2$ tension gradient after cardiopulmonary bypass. Asian Cardiovasc Thorac Ann. 2005; 13(3): 255-60.

15. Dubin A, Murias G, Estenssoro E, Canales H, Badie J, Pozo M, et al. Intramucosal-arterial $\mathrm{pCO}_{2}$ gap fails to reflect intestinal dysoxia in hypoxic hypoxia. Crit Care. 2002; 6(6): 514-20.

16. Lescano Alva C. Gases sanguíneos y trastornos ácido-base. Rev Peruana Med Intensiva. 2015; 5(3): 6-14.

17. Baigorri González F, Lorente Balanza JA. Oxigenación tisular y sepsis. Med Intensiva. 2005; 29(3): 178-84.

18. Ospina-Tascón G, Hernández G, Cecconi M. Understanding the venous-arterial $\mathrm{CO} 2$ to arterial-venous 02 content difference ratio. Intensive Care Med. 2016; 42(11): 4233-7.

19. He H, Long $Y$, Liu D, Wang $X$, Tang $B$. The prognostic value of central venous-to-arterial $\mathrm{CO} 2$ difference/arterial-central venous 02 difference ratio in septic shock patients with central venous 02 saturation $>80 \%$. Shock. 2017; 48(5): 551-7.

20. Ochagavia A, Baigorri F, Mesquida J, Ayuela JM, Ferrándiz A, García $X$, et al. Monitorización hemodinámica en el paciente crítico. Recomendaciones del grupo de trabajo de Cuidados Intensivos cardiológicos y RCP de la Sociedad Española de Medicina Intensiva, Crítica y Unidades Coronarias. Med Intensiva. 2014; 38(3): 154-69.

21. Vallée F, Vallet B, Mathe 0 , Parraguette J, Mari A, Silva S, et al. Central venous to arterial carbon dioxide difference: an additional target for goal-directed therapy in septic shock?. Intensive Care Med. 2008; 34(12): 2218-25.

22. Hernández Luna A, López Pérez H, Etulain González J, Olivera Guzmán C, Aguirre Sánchez J, Franco Granillo J. Delta de dióxido de carbono para valorar perfusión tisular como predictor de mortalidad en choque séptico. Med Crit. 2011; 25(2): 66-70.

23. Diaz del Castillo AH, Pérez Cely JA. Correlación entre el delta de CO2/Diferencia arteriovenosa con índice cardiaco en paciente en postoperatorio de cirugía cardiaca [Tesis]. Universidad de la Sabana: Colombia. Facultad de Medicina; 2011.

\section{Correspondencia:}

Washington Alfonso Trujillo Ulloa

Dirección: Av. Circunvalación N¹19, Urbanización Laderas del Norte. Chimbote, Perú.

Teléfono: 965616510

Correo electrónico: washi0910@hotmail.com

Recibido: 24 de octubre de 2020 Evaluado: 18 de febrero de 2020 Aprobado: 20 de febrero de 2020

( ) La revista. Publicado por Universidad de San Martín de Porres, Perú. (c) Br Licencia de Creative Commons Artículo en acceso abierto bajo términos de Licencia Creative Commons Atribución 4.0 Internacional. (http://creativecommons.org/licenses/by/4.0/)

ORCID iDs

Washington Alfonso Trujillo Ulloa ㄴ) https://orcid.org/0000-0002-8315-9943 José Elías Cabrejo Paredes ㄴ) https://orcid.org/0000-0002-7335-0541 